\title{
EL ESPACIO POR APRENDER, EL MISMO QUE ENSEÑAR: LAS URGENCIAS DE LA EDUCACIÓN GEOGRÁFICA
}

\author{
Marcelo Garrido Pereira*
}

\begin{abstract}
RESUMEN: La presente reflexión intenta indagar en las posibilidades del discurso pedagógico cuando se considera el saber espacial informal como un antecedente de prácticas transformativas. El saber espacial informal ha sido ampliamente negado por los currículos nacionales e incluso menospreciado por las apuestas didácticas que los profesores de geografía desarrollan. En tal sentido urge identificar las relaciones entre un saber que es experiencia y que es al mismo tiempo parte de un proyecto de actor y de sujeto en crisis. Con todo ello se proponen espacios de intervención pedagógica, categorías de síntesis de la experiencia espacial y caminos problematizadores para el desarrollo de una educación geográfica significante.
\end{abstract}

Palabras claves: Saberes geográficos informales. Reconstitución de la experiencia espacial. Espacios de intervención pedagógica.

\section{THE SAME SPACE FOR LEARNING AND TEACHING: THE URGENCIES OF GEOGRAPHY EDUCATION}

ABSTRACT: The present reflection questions the possibilities of the pedagogical speech when the informal space knowledge is considered as an antecedent of transformations practices. The informal space knowledge has been widely banned from the national curricula and is disdained in the didactic proposals developed by geography professors. It is thus urgent to identify the relations between a knowledge that is experience and a project of actor and subject in crisis. This leads us to propose pedagogical intervention spaces, synthesis categories of the space experience and problematizing ways to develop a significant geographic education.

Key words: Informal geographic knowledge. Reconstitution of the space experience. Spaces for pedagogical intervention

Candidato a doctor en Ciencias de la Educación, Pontificia Universidad Católica de Chile, profesor de geografía y licenciado en educación.E-mail: mgarridp@puc.cl 
El espacio por aprender, el mismo que enseñar: las urgencias de la educación...

\section{Espacio geográfico como experiencia}

$\mathcal{E}$ l espacio, como categoría conceptual, ha sido concebido de distintas formas dependiendo de la vertiente teórico-metodológica utilizada. Lo anterior, denota la existencia de un posicionamiento epistemológico, más o menos, conciente que ha estado a la base de los discursos geográficos. Sin embargo, pocas veces se intenta develar la estructura semántica de las construcciones conceptuales, tal vez por evidenciar un aparente consenso frente a la comunidad científico-técnica de una unicidad en la concepción de un objeto de estudio para la geografía. Sin entrar en el detalle de la problemática, interesaría aproximarse a la construcción de un objeto (o sujeto) de estudio que recupere ciertas nociones de los radicalismos socio-geográficos que permitan entender al "espacio geográfico" como un noción experiencial que es inherente a la vivencia de los sujetos, además de reconocer en ella una única esencia material-mental. Sin este ejercicio, resulta imposible situar cualquier contribución en el área de la educación geográfica, puesto que no se accede unívocamente a un sentido sobre el objeto de la geografía y por tanto no necesariamente nos referimos a una sola forma de enseñanza o a una sola forma de aprendizaje.

$\mathrm{Al}$ respecto resulta interesante rescatar aportaciones provenientes de la geografía humanística, específicamente aquellas realizadas por el profesor Yi-Fu Tuan quien sitúa al espacio, objeto de la geografía, en el plano de la experiencia:

Experiencia es un término que abarca las diferentes maneras a través de las cuales una persona conoce y construye la realidad. Estas maneras varían desde los sentidos más directos y pasivos como el olfato, paladar y tacto, hasta la percepción visual activa y la manera indirecta de simbolización (...). Los espacios del hombre reflejan la calidad de sus sentidos y su mentalidad. La mente frecuentemente extrapola más allá de la evidencia sensorial. (Tuan, 1983, p. 9 y 18)

El espacio, como noción de soporte, es un concepto limitante para comprender la experiencia del hombre. Esta última no actúa sobre el espacio, sino que más bien lo configura. Todo ello es esencial para situar un contenido educativo como lo es el espacio, pues lo amplia al campo de lo cotidiano, de lo inherente, y de lo nuclear. Tal es la complejidad de este espacio, que supera todo orden estrictamente material, al mismo tiempo que no se agota en lo fijo: 
Espacio es un término abstracto para un conjunto complejo de ideas. (...) Sin embargo, existen ciertas semejanzas culturales comunes, y ellas se fundamentan básicamente en el hecho de que el hombre es la medida de todas las cosas. En otras palabras, los principios fundamentales de la organización espacial se encuentran en dos tipos de hechos: la postura y la estructura del cuerpo humano y las relaciones (sean próximas o distantes) entre personas. El hombre, como resultado de su experiencia íntima con su cuerpo y con otras personas, organiza el espacio a fin de adaptarlo a sus necesidades biológicas y relaciones sociales. (Idem, ibid., p. 39)

El espacio recupera su raíz idealista en tanto, no es un objeto realmente acabado fuera de uno, más bien se presenta como un posibilidad de construcción histórica de si mismo. No está en tanto cosa fuera de mi experiencia, no existe en cuanto si, sino para mi y por mi:

La experiencia de espacio y tiempo es principalmente subconsciente. Tenemos un sentido de espacio porque podemos movernos y de tiempo porque, como seres biológicos, pasamos fases recurrentes de tensión y calma. El movimiento que nos da el sentido de espacio es en sí mismo la solución de la tensión. (Idem, ibid., p. 132)

Este mismo espacio, objeto (sujeto) disciplinar, es trabajado por la geografía crítica. Las múltiples aportaciones nos sitúan en la radical forma de comprenderlo más allá de su desmantelación en variables. Un buen ejemplo de ello, son las aportaciones de Milton Santos quien posibilita la comprensión del espacio en tanto totalidad de los sistemas de fijos y flujos que lo configuran:

El espacio es la materia trabajada por excelencia. Ninguno de los objetos sociales tiene una imposición tan grande sobre el hombre, ninguno está tan presente en lo cotidiano de los individuos. La casa, el lugar de trabajo, los puntos de encuentro, los caminos que unen esos puntos, son igualmente elementos pasivos que condicionan la actividad de los hombres y rigen la práctica social. La praxis, ingrediente fundamental de la transformación de la naturaleza humana, es un dato socio-económico, pero es también tributaria de los imperativos espaciales. (Santos, 1996b, p. 28)

En dichas aportaciones se refleja además, el carácter condicionante de la configuración espacial. El espacio es resultado de una construcción de experiencia, al mismo tiempo que condiciona la dinámica posterior de la misma. El objeto de la geografía, se reafirma en lo significativo al mismo tiempo que en lo histórico; se amplia en su sentido material al 
El espacio por aprender, el mismo que enseñar: las urgencias de la educación...

mismo tiempo que mental; se potencia en lo fijo y en lo dinámico; se educa en relación a si mismo y a los demás.

\section{La educación del espacio geográfico y el proyecto de un sujeto}

La configuración de un espacio geográfico como la proyección de lo social permite comprender a la experiencia social en tanto sitio, además que situación. El espacio geográfico, ya no se entiende más como un receptáculo, sino que como el resultado de una multiplicidad de interacciones entre los seres humanos y los contextos socio-naturales, toda vez que la movilidad de flujos permite la dinámica de los aparentes fijos. En el ámbito de la evolución de las ideas y de las representaciones de lo social, este espacio geográfico es cada vez menos un espacio para la construcción de subjetividad y cada vez menos un espacio para la construcción de socialización, toda vez que se asiste al desmembramiento de la idea de una sociedad única, y emerge el individuo como categoría aislada de un proyecto colectivo. Este espacio geográfico representa, cada vez más, un espacio de construcción de una experiencia desvinculada de si mismo y de los demás. Este espacio geográfico que es ahora una experiencia, por sobre todo, individual, plantea unos desafíos educativos, en tanto la enseñanza y el aprendizaje espacial en la escuela aparecen desconectados de un proyecto de sujeto. Algunos antecedentes que nos permiten referirnos a esta cuestión de la experiencia que no se ancla a un proyecto, en el presente caso, educativo, la encontramos en la Sociología de la Experiencia Educativa:

La fabricación de actores y de sujetos no surge ya armoniosamente del funcionamiento regulado de una institución en la cual cada uno desempeña un rol. Entonces es necesario reemplazar la noción de rol por la de experiencia. Los individuos ya no se forman solamente en el aprendizaje de roles sucesivos propuestos a los estudiantes, sino en su capacidad para manejar sus experiencias escolares sucesivas. (Dübet y Martucelli, 1998, p. 14)

La experiencia total y compleja de los individuos ha sido obviada por los sistemas formalizados de enseñanza, al tiempo que se ha impedido que la escuela actúe como espacio para potenciar la subjetivación y la socialización de los protagonistas de la educación. Cada vez menos, la escuela y los espacios formalizados intentan indagar en la experiencia del 
“otro" y cada vez más intentan anular la diferencia por medio de prácticas homogeneizantes. Una de las dimensiones de esa experiencia diferencial con que los estudiantes llegan, es la dimensión espacial, vale decir, aquella que dice relación con la actuación de un sistema indisociable de significaciones y materialidades. El aprendiz manifiesta de diversos modos la imposibilidad de construir, en diálogo, su propia experiencia que se ancla a su vivencia cotidiana: "A tal punto son diversos los públicos escolares, a tal punto las situaciones escolares parecen poco reguladas que a veces podemos tener el sentimiento de que los alumnos construyen al lado o en contra de la escuela" (Dübet y Martucelli, 1998, p. 15).

En este sentido, y frente a las manifestaciones de resistencia experiencial, cabría destacar que el sujeto intenta, diferencialmente construir subjetividad y establecerse como sujeto autónomo, tarea un tanto compleja si se asume que la relación pedagógica se manifiesta como un acto asimétrico, de intereses superpuestos y con protagonistas diversos. Con todo ello, la experiencia compleja o total (ver la similitud con el concepto de trama de Paulo Freire), o de modo específico, la experiencia espacial se encuentra íntimamente relacionada con la efectividad del proyecto de construcción de sujeto y es un acto, la mayor parte de las veces, con objetivos transformativos que no deben seguir siendo desconocidos por los enseñantes en su labor pedagógica:

Los actores no se identifican únicamente con sus pertenencias y sus intereses, se definen también como sujetos, no a través de un decreto de libertad, sino porque la vida social propone una representacion del sujeto. Significa que ellos se definen también por su creatividad, por su autonomía, por su libertad, por todo lo que, paradójicamente se presenta como no social .(Dübet y Martucelli, 1999, p. 79)

En este contexto cabría preguntarse cómo se potencia una enseñanza-aprendizaje que pueda contribuir a la construcción de un proyecto de sujeto, que sea también un proyecto de vivencia espacial. Una posible solución es no desconociendo su experiencia en el acto educativo. Esto implica valorar la experiencia compleja y total y develar la ocultación de los procesos de subjetivación que surgem de la dominación experiencial de los sujetos.

La enseñanza aparece entonces, como depositaria de una de las labores más dificultosas, pues permitiría romper con la idea de "los individuos" como categoría constante y potenciaría la construcción de las sub- 
El espacio por aprender, el mismo que enseñar: las urgencias de la educación...

jetividades que dan origen y sentido a la actuación personal. El enseñante ya no sólo se instala en la posibilidad de construcción de si, sino que con su actuación, acompaña la construcción de otros, a través de una pedagogía por sobre todo dialogante que incita a la búsqueda y anima en la espera:

El maestro es aquel que ultrapasó la concepción de una verdad como fórmula universal, solución y resolución del ser humano, para elevarse a la idea de una verdad como búsqueda. El maestro no posee la verdad y no admite que alguien pueda poseerla. Le horroriza el espíritu de propietario del pedagogo y su seguridad en la vida. (Gusdorf, 1963, p. 239)

La enseñanza tiene, en este sentido, un compromiso más con la verdad de los individuos que intentan y añoran la construcción de su subjetividad, que con el currículo nacional, el sistema formal y las verdades reveladas por las instituciones educativas locales, sectoriales o nacionales. La semántica de las verdades reveladas es uno de esos puntos en que se establece algún grado de varianza en la construcción de un conocimiento y una identidad profesional-pedagógica, y es por tanto desde donde emergen los condicionamientos del contenido pedagógico. El espacio como selección cultural y como contenido a condicionar, ha sido desde siempre una verdad revelada en tanto permite ajustarnos a un convención cartográfica, o a una nomenclatura espacial básica, o a un taxón, o a cualquier otro tipo de no experiencia que aleja al estudiante de nuestros discursos pedagógicos, al mismo tiempo que no logramos establecer relaciones de significantes entre nuestro saber en uso y sus saberes cotidianos sobre el espacio:

La cultura no es otra cosa sino la toma de conciencia, por cada individuo, de esa verdad que hará de él un hombre. El pedagogo asegura lo mejor posible enseñanzas diversas; reparte conocimientos. El maestro quiere ser antes que todo iniciador de la cultura. La verdad es para cada uno el sentido de su situación. (Gusdorf, 1963, p. 241)

En la construcción siempre riesgosa de nuestro conocimiento y de nuestra identidad profesional-pedagógico-geográfica, las posibilidades de establecer una reflexión sobre los mecanismos de aprendizaje en el contexto de la construcción de proyecto de sujeto, son siempre amplias. El aprendizaje se instala como construcción de significados, en tanto se amplían las capacidades para estructurar la experiencia en torno a una meta 
cognitiva. No hay aprendizaje, sin significados, pero tampoco hay aprendizaje sin una meta sobre el conocimiento. Es justamente en este sentido, que aparece como prioritario el aprendizaje del espacio, en tanto remite a metas cognitivas que el individuo considera necesarias para la construcción de su subjetividad. El aprendizaje es visto en relación a cada una de las vivencias, y por tanto se supera la idea del cumplimiento de logros en función del desarrollo biológico. Los estadios de aprendizaje se encuentran situados, y su progresión ha sido estancada cuando se ha intentado dinamizarla en base a la no autonomía:

En el hombre adulto son extremamente complejos los sentimientos e ideas relacionados con espacio y lugar. Se originan de las experiencias singulares y comunes. No obstante cada persona comienza como un niño. Con el tiempo, del confuso y pequeño mundo infantil, surge la visión del mundo del adulto, subliminalmente también confusa, mas sustentada por las estructuras de la experiencia y del conocimiento conceptual. (Tuan, 1983, p. 22)

Está demás decir que el aprendizaje, en este caso, espacial, se construye en base a una autonomía relativa, pues, el individuo no sólo construye subjetividad, sino que además se encuentra en la compleja tarea de avanzar en su socialización. Para ilustrar lo anterior, cabría destacar uno de los ejemplos utilizados por el profesor Yi-Fu Tuan para comprender el aprendizaje espacial:

A medida que el niño crece, se va apegando a objetos, en lugar de apegarse a personas importantes, y finalmente a localidades. Para el niño, lugar es un tipo de objeto grande y un tanto inmóvil. (...) El horizonte geográfico de un niño se expande a medida que él crece, pero no necesariamente paso a paso en dirección a la escala mayor. Su interés y conocimiento se fijan primero en la pequeña comunidad local, después en la ciudad, saltando el barrio; y de la ciudad su interés puede dirigirse para la nación y para lugares extranjeros, saltando la región. (Tuan, 1983, p. 33 y 35)

El aprendizaje del espacio fortalece la construcción de sujeto, en tanto se potencia lo cotidiano como categoría configuradora de si mismo. Pese a ello, el aprendizaje del espacio no sólo tiene que ver con lo cotidiano, entendido como lo más próximo. De hecho puede resultar que una experiencia espacial se constituya por fenómenos con expresiones escalares distintivas. El aprendizaje espacial no se realiza, necesariamente, desde la fragmentación de las escalas de afectación, como la mayor parte de las ve- 
El espacio por aprender, el mismo que enseñar: las urgencias de la educación...

ces hemos intentado propiciarlo. El aprendizaje espacial tiene que ver con la complejidad de una subjetividad que se construye por la vinculación de las afectaciones distintivas en su extensión e intensidad.

Es imposible discutir el espacio experiencia sin introducir los objetos y los lugares que definen el espacio. El espacio del niño se amplía y se torna más bien articulado a medida que él reconoce y alcanza más objetos y lugares permanentes. El espacio se transforma en lugar a medida que adquiere definición y significado. (Idem, ibid., p. 151).

Ese mismo principio, rescatado por el socio-constructivismo en los discursos pedagógicos, es un principio bastante olvidado en la geografía. La experiencia espacial evoluciona y progresa en la medida en que se es capaz de identificar (acto a ser potenciado vía aprendizaje escolar) los significados, no como entidades aisladas, sino como condiciones de esencia de los significantes. La dinámica de la experiencia espacial encuentra su progresión en la búsqueda de una sustancia ocultada, original y compleja y que tiene que ver en última instancia con lo que represento sobre mi.

\section{La educación del espacio geográfico y el proyecto de un actor}

El proyecto de sujeto no se encuentra desligado de la construcción de integración a lo social. Los individuos que avanzan en la construcción de subjetividad, normalmente lo hacen avanzando también, en el plano de la socialización. Esta dualidad que no es contradictoria sino complementaria, no hace sino complejizar la apuesta educativa, pues la enseñanza y el aprendizaje debieran estar orientados al acompañamiento experiencial que todo individuo intenta desarrollar en tanto se define como tal y en relación con otros. De modo específico, la construcción de una integración es el camino por medio del cual los individuos se transforman en actores de lo social. Lo hacen construyendo significados, adhiriendo a ciertas pautas ya pre-establecidas y dejándose, hasta cierto punto, normar por un habitus pre-existente. Al respecto la Sociología de la Experiencia Social nos señala:

Cada uno de nosotros actúa en función de un principio de integración definido como la interiorización de lo social (...) nuestra personalidad está fuertemente constituida por nuestra adhesión subjetiva a las expectativas sociales adquiridas en el curso de las fases de socialización prima- 
ria... nuestra historia construyó una segunda naturaleza que está tan arraigada en nosotros como una primera (...) pero esa segunda naturaleza no es solamente un ser dado una vez por todas, es además una actividad ya que la actuamos y la actualizamos permanentemente en el curso de los encuentros y de los desafíos de nuestra vida. (Dübet y Martucelli, 1999 , p. 77 y 78 )

El devenir de las experiencias sólo puede ser manejado y/o gestionado sobre la lógica de una actuación que se aprehende y se aprende. En este sentido la integración de los sujetos se produce no tan sólo por la subordinación, sino por el diálogo entre lo establecido socialmente y su propias ideas sobre si mismo, así como por las posibilidades de resistencia. En este sentido la educación aparece como un proceso vital en tanto intenta potenciar una aparente crisis que surge de la desvinculación y anomia de los individuos respecto de un nuevo orden social. El enseñante no interacciona neutralmente con el aprendiz. En muchas ocasiones aparecerá enfrentándose, cediendo, normalizando, sin embargo no debiera olvidar que una de sus tareas pedagógicas consistirá en acompañar al aprendiz en un proceso de socialización, que muchas veces tiende a la simetría: "Los actores se socializan a través de estos aprendizajes y se constituyen como sujetos en su capacidad de manejar su experiencia, de devenir, por una parte, en autores de su educación" (idem, 1998, p. 14).

En el contexto de la enseñanza, la construcción del mundo de la vida es una tarea que implica más allá que una mera construcción de mis tierras incógnitas personales. Se trata ahora de situarme como "con otros" configuradores de una experiencia espacial que incluye. El principio de un espacio que no es la mera suma, ni efecto perverso de agregación de experiencias. Se trata más bien de un espacio construido en base a renuncias concientes y concurrencias libres. Se trata de un espacio de actores, en donde los compromisos y la adhesión generan integración social. Contenido desafiante desde la enseñanza, pues implica recuperar la idea de un proceso educativo que es ante todo negociación y que propugna por cierto, la simetría del acto pedagógico. En este sentido, Paulo Freire nos habla de cómo los individuos toman su vida como objeto de construcción y levantan desde allí, categorías reflexivas:

No puedo comprender a los hombres y a las mujeres más que simplemente viviendo, histórica, cultural y socialmente existiendo, como seres que hacen su camino y que, al hacerlo, se exponen y se entregan a ese 
El espacio por aprender, el mismo que enseñar: las urgencias de la educación...

camino que están haciendo y que a la vez los rehace a ellos también (...) A diferencia de los otros animales, que no llegaron a ser capaces de transformar la vida en existencia, nosotros, en cuanto existentes, nos volvimos aptos para participar en la lucha y defensa de la igualdad de posibilidades por el hecho mismo de ser, como seres vivos, radicalmente diferentes unos de las otras y unas de los otros. (Freire, 1993, p. 93)

La enseñanza no neutral que desmitifica la asepsia del acto pedagógico es un primer paso para instalarse en la posibilidad de construir una realidad en torno al diálogo democrático. Es esta enseñanza, posicionada, la que debe propiciar, además, la recuperación de aquellos saberes que hemos considerado inválidos, poco rigurosos, asistemáticos, vulgares, y de sentido común. La democracia en el acto educativo se garantiza desde el reconocimiento del otro diferente, que es también otro "espacial" diferente. Urge vitalizar como contenidos aquellos prácticas espaciales que son consustanciales a los protagonistas de la educación y que se mantienen al margen, y más aún excluidas de toda posible valoración colectiva. No se es más un ocupador de rol, sino un constructor de experiencia en diálogo y en respeto:

No puede haber camino más ético, más verdaderamente democrático, que revelar a los educandos cómo pensamos, las razones por las cuales pensamos de tal o cual forma, nuestros sueños, los sueños por los que luchamos, dándoles al mismo tiempo pruebas concretas, irrefutables, de que respetamos sus preferencias aunque sean opuestas a las nuestras. (Freire, 1996, p. 43)

En el contexto del aprendizaje aparece sugerente indicar que todo intento por explorar conceptuar, estructurar o aplicar conocimientos, pareciera ser una tarea de corte apriorística en tanto se presupone que el origen del mismo se encuentra en la interacción de lo dado como externo a si (primera naturaleza) y aquello que es interno a mi, como en una relación de opuestos con simetría en sus fuerzas. Sin embargo, se debe reconocer que en el origen de esos conocimientos que serán aprendidos, media por sobre todo la intencionalidad sobre una primera naturaleza. Es el ser humano quien se aproxima al objeto-sujeto potencialmente cognitivo y lo dado como externo a así no concurre libremente a la experiencia. El conocimiento no puede ser, sino entendido como una segunda naturaleza, como una construcción racional-mental-corporea que ha surgido de la intención de conocer. Al respecto algunas ideas de Milton Santos nos refieren a esta problemática: 
La naturaleza siempre fue la despensa del hombre, incluso cuando esta se hallaba en su fase pre-social. Pero para que el animal hombre se convierta en el hombre social es indispensable que pase a ser además el centro de la naturaleza. Lo que se consigue mediante el uso conciente de los instrumentos de trabajo. En ese momento, la naturaleza deja de ser un simbiosis entre el trabajo del hombre y una naturaleza cada vez más modificada por ese mismo trabajo. (Santos, 1990, p. 177)

Las formas, pues, tienen un papel en la realización social. (Santos, 1996b, p. 28)

En este sentido, los aprendizajes son intencionados ya no sólo desde los intereses de los estudiantes, sino además desde los intereses del profesor. Los aprendizajes espaciales han estado históricamente situados en relación a su origen cognitivo. Hemos dado como hecho, que en el contexto de una disciplina puente, hay saberes que sólo se obtienen acudiendo a ellos por medio de la experimentación, asumiendo que son, en la medida de ellos mismos. Este es una dato del contexto de las prácticas pedagógico-geográficas, que niega la segunda naturaleza, característica de la experiencia espacial. No hay aprendizaje sino es en la medida de si mismo, sino es en la medida de nuestros significados, sino es en la medida de nuestras intencionalidades, sino en la medida de las materialidades. La geografía escolar no asume su condición de ciencia experiencial, más que experimental, tal vez por el miedo a no ser reconocida como un saber válido al interior de los currículos de nuestros países. La formación de individuos en torno a unos proyectos de sujeto o unos proyectos de actor, no se desvincula del aprendizaje de la experiencia espacial, en tanto esta última es expresión total y compleja de lo social:

El contenido corporificado, al ser transformado en existencia, es la sociedad incorporada a las formas geográficas, la sociedad transformada en espacio. La fenomenología de Hegel sería la transformación de la sociedad total en espacio total. La sociedad sería el ser; y el espacio, la existencia. El ser se metamorfosea en existencia por mediación de los procesos impuestos por sus propias determinaciones, las cuales hacen aparecer cada forma como una forma contenido, un individuo separado capaz de influenciar el cambio social. Es un movimiento permanente, $y$, por ese proceso infinito, la sociedad y el espacio evolucionan contradictoriamente. (Santos, 1996b, p. 28)

Estas palabras de Milton Santos interpelan a la necesidad de entendimiento de una experiencia espacial inacabada y potencial, dinámica a la 
El espacio por aprender, el mismo que enseñar: las urgencias de la educación...

vez que permanente, resultado parcial a la vez que condicionamiento habitusial. La tarea siguiente es el intento de comprensión de las posibilidades que tiene la experiencia espacial de ser re-construida por medio de las interacciones educativas.

La reconstrucción de la experiencia espacial: el papel de la educación geográfica

\section{La síntesis de la experiencia espacial}

En el contexto de un educación geográfica que se desarrolla intentando fortalecer la subjetivación y la socialización de los individuos, no cabe sino esperar una varianza en los escenarios de intervención didácticopedagógica. Se trata de desarrollar un proceso de enseñanza-aprendizaje del espacio geográfico en diálogo con las experiencias espaciales diferenciales que convergen en el aula. A modo de propuesta convendría realizar una pequeña sistematización de las posibilidades de convergencia de geografías en el aula, a tener en cuenta, para intentar desarrollar una pedagogía geográfica más democrática. Se considera para tal caso la relación de concurrencia libre entre los aprendices con sus proyectos de socio-subjetivación y los enseñantes con su reconocimiento del otro experiencial:

\section{Cuadro 1}

(La educación geografía en situación geográfica)

Cohesión de Proyecto socio-subjetivo del Aprendiz

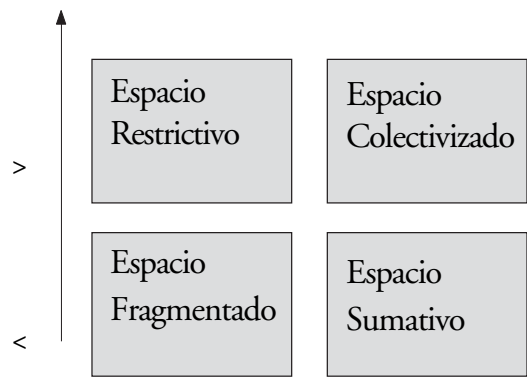

$<\quad>$ Reconocimiento de la experiencia por parte el enseñante 
En el cuadro expuesto, se visualiza la conjugación de situaciones de concurrencia experiencial, sin las cuales se hace dificultoso planificar un proceso de enseñanza-aprendizaje. De modo específico, se intenta situar a la geografía en sí misma y en relación, tanto a los proyectos de sociosubjetivación de los aprendices, como a los proyectos de intervención pedagógica de los enseñantes. Se trata de una trama esquematizada en donde convergen diferencialmente las vivencias de los protagonistas educativos en torno a dos ejes. El primero de ellos, el de cohesión de los proyectos socio-subjetivos de los aprendices, dice relación con el grado de coherencia, concordancia y enlace entre los mecanismos de subjetivación y de socialización puestos en acción por el individuo para el desarrollo de su dimensión sujeto-actuante. Esa cohesión entra en juego pedagógico, toda vez que se ve enfrentada a la propuesta de intervención pedagógica, que reconoce diferencialmente estos proyectos, como experiencias legítimas de ser consideradas en la construcción de un conocimiento compartido.

Donde hay concurrencia de un bajo reconocimiento de la experiencia del otro por parte del enseñante, además de proyectos socio-subjetivos vagamente cohesionados tenemos un enmarcamiento experiencial que podríamos denominar espacio fragmentado para la interacción pedagógica. Si por el contrario tenemos un alto reconocimiento de la experiencia de los aprendices, así como un cohesionado proyecto de sujeto-actor, el enmarcamiento experiencial, podría ser denominado espacio colectivizado, vale decir, un espacio compartido, en donde la interacción pedagógica se encuentra construyendo relaciones democráticas y liberadoras, en tanto se asume la tarea conjunta con intenciones compartidas y con aportes complementarios.

Donde hay concurrencia de un bajo reconocimiento de la experiencia del otro, pero una alta cohesión de los proyectos socio-subjetivos por parte de los aprendices, tenemos una situación de subvalorización que repercute en la sustracción de las vivencias que no se ajustan a la planificado desde la intervención pedagógica. Este es un espacio restrictivo, en tanto se resta la capacidad del individuo como generador de su propia actuación y de su propia subjetividad en el proceso educativo. Al contrario, donde hay un alto reconocimiento por parte del enseñante, respecto de la experiencia del otro, al mismo tiempo que convergen individuos aprendices con un muy poco cohesionado proyecto socio-subjetivo, tenemos un espacio de simple agregación, en donde la dinámica de concurrencia es más bien un efecto perverso, que no implica comu- 
El espacio por aprender, el mismo que enseñar: las urgencias de la educación...

nión en algún único proyecto de construcción de conocimiento, ni tampoco implica intencionalidades compartidas. Se esta enfrente de un enmarcamiento que apela al establecimiento de vínculos y relaciones, metas y proyecciones colectivas. Se trata de un Espacio Sumativo.

El enmarcamiento de la intervención pedagógica, en esta geografía de la experiencia educativa, es una situación que se puede transformar. En este sentido, no hay educación geográfica que no se encuentre situada, al mismo tiempo que no hay educación geográfica que no tenga la potencia de transformarse a si misma. Este enmarcamiento de la intervención pedagógica viene unido indisociablemente al posicionamiento teórico antes enunciado, y también a la necesidad metodológica de recuperar alguna categoría semantizadora del espacio geográfico, en tanto permite privilegiar ciertas dimensiones de la experiencias espaciales que concurren en el acto educativo. Las categorías semantizadoras del espacio geográfico han aparecido en el foco de la discusión, en tanto, tienden a ser confundidas como un problema de indefinición del objeto de estudio. De este modo, territorio, lugar, región, paisaje y más recientemente geosistema y medio-ambiente, han aparecido como conceptos compitiendo entre sí, en esta carrera por establecer definitoriamente aquello que es exclusivo como objeto de los profesionales de la geografía. Pese a las diferencias establecidas entre dichos conceptos, nadie podría discutir, su sinonimia con el concepto de espacio geográfico, vale decir, en el contexto de la reconstitución de la experiencia espacial, las categorías semantizadoras del espacio geográfico representan una opción metodológica de comprensión y entendimiento del mismo. En tal sentido y considerando la apuesta teórico-metodológica del presente ensayo, se podría afirmar que todos esos conceptos remiten a una única experiencia espacial y que se constituyen como categorías conceptuales de síntesis. La síntesis potencial encuentra argumento, considerando que toda actuación en la configuración de una idea de totalidad espacial, coincide con una experiencia que "yo" concuerdo declarar como una única forma de sentido. Al respecto, resultaría interesante re-leer un ejercicio pedagógico propuesto por la profesora María Raquel Pulgarín (2003) en donde hace referencia a estas categorías pero desde sus potencialidades analíticas, entendiendo que su apuesta didáctica concibe un aprendizaje desde la desagregación de la mutidimensionalidad del espacio geográfico.

Con todo ello se podría afirmar, que no hay reconstrucción de la experiencia espacial en diálogo pedagógico, sino se potencian las formas de sentido (significaciones) que cada individuo (más o menos actor; más o 
menos sujeto) ha dado a su propia experiencia. A modo de ejemplo, mientras un profesor utiliza al paisaje como categoría analítica, la oferta pedagógica intentará reducir su experiencia a unas categorías ajenas a su vivencia. La apuesta de esta reflexión, es considerar al paisaje, y a las otras categorías, como conceptualizaciones que tienden a la síntesis de la experiencia. Se trata ahora, de concebir estas semantizaciones del espacio geográfico, como mecanismos de reconstrucción de significados, situados históricamente y pertenecientes a un proyecto sobre si mismo y sobre un nosotros.

\section{Cuadro 2}

(Relaciones de significado que permiten la síntesis de la experiencia espacial)

\begin{tabular}{|c|c|c|c|}
\hline Categoría & Relación Significada & Categoría & Relación Significada \\
\hline Paisaje & $\begin{array}{l}\text {-Transtemporalidad } \\
\text {-Acumulación desigual } \\
\text { de los tiempos } \\
\text {-Morfología Perceptiva } \\
\text {-Belleza constitutiva }\end{array}$ & Región & $\begin{array}{l}\text {-Unicidad } \\
\text {-Particularidad } \\
\text {-Límite } \\
\text {-Integración }\end{array}$ \\
\hline Territorio & $\begin{array}{l}\text {-Política } \\
\text {-Gestión } \\
\text {-Poder } \\
\text {-Ideología } \\
\text {-Ordenamiento }\end{array}$ & Medio Ambiente & $\begin{array}{l}\text {-Soporte } \\
\text {-Circunstancias } \\
\text {-Entorno-Contorno } \\
\text {-Hábitat } \\
\text {-Ethos }\end{array}$ \\
\hline Lugar & $\begin{array}{l}\text {-Arraigo } \\
\text {-Pertenencia } \\
\text {-Filiación } \\
\text {-Simbolismos } \\
\text {-Identidad }\end{array}$ & Geosistema & $\begin{array}{l}\text { Estructura } \\
\text { Funcionamiento } \\
\text { Complejidad } \\
\text { Homeostasia } \\
\text { Retroalimentación }\end{array}$ \\
\hline
\end{tabular}

Aun cuando dichas categorías posibilitaron la generación de múltiples discursos geográficos, hoy emergen como las posibilidades de reconstitución de la experiencia espacial, en tanto permiten dimensionar la configuración del espacio en torno a dimensiones perceptivas y representativas. 
El espacio por aprender, el mismo que enseñar: las urgencias de la educación...

Perceptivas en tanto cognición compleja de las interrelaciones que se desarrolla a través de los sentidos; y Representativas, en tanto cognición compleja de las interrelaciones, que se desarrolla a través de las significaciones. El educador en geografía debiera posibilitar la autonomía del "otro", en tanto intenta re-constituir su propia experiencia espacial, de acuerdo con lo que considera significante, propio y cognitivamente concordante respecto de sus vivencias cotidianas. Las categorías de síntesis permiten reducir complejidad, sin fragmentar la experiencia, en tanto se asume como recurso metodológico básico para re-construirse a si mismo.

\section{La problematización de la experiencia espacial}

La cooperación en el aprendizaje, no es sino una construcción de un contexto colaborativo, un paisaje de posibilidades, un espacio adecuadamente ambientalizado. El proceso de enseñanza-aprendizaje recupera la humanidad de su naturaleza, al reposicionar a los sujetos como creadores y transformadores de su realidad individual y colectiva, partiendo de la rotura de la asimetría implícita en dicho proceso y asumiendo para este la esencia de una olvidada dialéctica. Como si todo ello no fuese suficiente, la toma de conciencia de las potencias colaborativas armoniza y estabiliza los ambientes de aula, pues los transforma justamente en realidad diferenciada y no totalizada. La fragmentación del sujeto-contexto no se puede sostener desde esta perspectiva y por tanto la ausencia de responsabilidades educativas pasa a constituirse en una mentira lo suficientemente "ingenua" como para destacarla.

Estos elementos socio-constructivistas nos permiten re-plantear los escenarios educativos. La experiencia espacial se encuentra como manifestación de la situación psico-socio-colaborativa, pero al mismo tiempo, dicha experiencia espacial es re-construida considerando la experiencia compleja de los protagonistas educativos. La geografía potencia la construcción de espacios de aprendizaje y se nutre de dichos espacios para hacer significativas las apuestas pedagógicas. En el contexto de constituir una propuesta de discurso didáctico-pedagógico para la re-construcción de la experiencia espacial, ya no bastaría sólo con la adopción de un cuerpo teórico que sitúe al enseñante respecto del objeto de estudio geográfico, ni con la sola identificación de un espacio de intervención, ni tampoco bastaría con proponer una categoría de síntesis, sino que además debería problematizarse la experiencia en torno a algunos ejes de importancia para la educación en geografía. La literatura 
geográfica ha definido la reconstrucción de la "idea” de espacio proponiendo una serie de categorías problematizadoras, de entre las cuales, hemos considerado pertinente destacar algunas, dado su congruencia con la metas pedagógicas de formación de un sujeto-actor: la interrelación, la escala y la conciencia. La interrelación haciendo referencia a la dimensión conceptual que debiera promover la educación geográfica; la escala situándose en el plano de lo procedimental que configura la educación para la re-construcción de una experiencia espacial; y la conciencia para recuperar las posibilidades de actuación-transformación de la misma experiencia.

\section{Lo conceptual en la experiencia espacial}

La esencia primaria que emerge de los discursos geográficos revela la importancia de las interrelaciones en la configuración de la experiencia espacial. La educación geográfica acude con reiteración a la fragmentación del saber en un intento metodológico por abordar las principales dimensiones del espacio, con el consecuente descuido por re-constituir la experiencia. El famoso puente curricular aparece más bien como un proyecto de largo plazo que nadie concretiza y los intentos por hacerlo, más bien se reducen a ilustrar posibles conexiones, interacciones, etc. La geografía no sólo potencia el análisis de la experiencia, sino que también la síntesis. Es esta última cualidad potenciada la que retorna al proyecto de un único sujeto capaz de entenderse como todo complejo. El desafío problematizador que emerge es construir una educación geográfica que revele como principal contenido conceptual "la interrelación". En este sentido, la búsqueda de espacios relacionales genera cementación de la aparente esquizofrénica realidad espacial que les presentamos a nuestros estudiantes. El hacer para si, un concepto, que es indispensable para articular las lógicas de acción de los sujetos, permite avanzar en ser para otros. La interrelación te posiciona en un mundo de interacciones con sentido, toda vez que se avanza en el reconocimiento y entendimiento del sentido de las responsabilidades. Algo de ello queda reflejado en la propuesta de reforma del pensamiento que está a la base del discurso de Edgar Morin:

En efecto, existe complejidad mientras sean inseparables los componentes diferentes que constituyen el todo (como lo económico, lo político, lo sociológico, lo psicológico, lo afectivo, lo mitológico) y haya un tejido interdependiente, interactivo e interretroactivo entre las partes y el todo, el todo y las partes. (Morin, 2000, p. 14) 
El espacio por aprender, el mismo que enseñar: las urgencias de la educación...

El debilitamiento de una percepción global conduce al debilitamiento del sentido de responsabilidad, ya que cada uno tiende a no ser responsable más que de sus tareas especializadas, así como al debilitamiento de la solidaridad, porque nadie percibe su lazo orgánico con su ciudad y sus conciudadanos. (Idem, ibid., p. 20)

La interrelación permite configurar una arquitectura de la experiencia espacial, que es además una reinvención sobre si mismo y su relación con el todo. Se trata de avanzar en la dimensión creativa y re-configurativa de la experiencia espacial, toda vez, que es un juego expresivo por encontrar y resignificar las partes, además de ser juego de astucia y perseverancia por encontrar el pegamento o la filiación adecuada. La educación geográfica debe proporcionar el acompañamiento adecuado en la comprensión de la interrelación, asumiendo que es ella quien deberá responder a propósito de la fragmentación de los espacios personales y de la pérdida de la solidaridade entre esos mismos espacios. Cabría destacar además que la cimentación de lo parcelado se potencia relevando algunas dimensiones de la experiencia interrelacional. Un buen ejercicio sería por ejemplo utilizar como pegamento las significaciones que están a la base de las ya señaladas categorías de síntesis geográficas, sin con ello decir que la suma de los saberes fragmentados es igualitario o equivalente a la experiencia espacial compleja: "Todo conocimiento constituye a la vez una traducción y una reconstrucción, a partir de señales, signos, símbolos, bajo la forma de representaciones, ideas, teorías, discursos" (idem, ibid., p. 29).

A este respecto, la adopción de la interrelación como categoría problematizadora-conceptual de la educación geográfica, implica asumir que no hay geografía mientras no se construyan o re-construyan esos espacios interrelacionales. No hay por tanto desarrollo de habilidades o destrezas cognitivas, ni desarrollo de contenidos, específicamente geográficos, si no se ha producido la re-configuración de la experiencia espacial. El aprendizaje espacial es aquel que se articula en torno a la síntesis y no necesariamente al mero análisis. La apuesta pedagógico-geográfica que sólo utiliza el análisis, la reducción o la parcelación como competencias a desarrollar, es una apuesta anti-geográfica. En este mismo sentido, cobran especial importancia las definiciones sobre los objetivos geográficos que debieran estar presentes en toda planificación pedagógica. Todo ello, porque existiría el riesgo siempre constante, de no culminar una enseñanza geográfica o no provocar un aprendizaje espacial. Un antecedente de ello es la limitación de los instrumentos de evaluación y calificación que utilizamos para defi- 
nir qué aprendió sobre el espacio. ¿ Existe, de acuerdo a la secuenciación de contenidos y objetivos, la posibilidad siempre cierta de verificar un aprendizaje espacial? Esta y otras preguntas apelan a la continuidad que debiera tener el proceso formativo-geográfico que no se reduce a algunas horas en unos cuantos niveles, pues sobrepasa incluso el ámbito formal. Lo complejo de este asunto, sin embargo, no debiera detener la motivación para actuar pedagógicamente. La tarea siguiente es hacernos cargo de lo esencial de nuestra disciplina, en el entendido de que sabemos que su esencia es una herramienta para la transformación social de este mundo diaclasado.

\section{Lo procedimental en la experiencia espacial}

La escala como aspecto procedimental a trabajar para la re-construcción de una experiencia espacial, no tiene que ver exclusivamente con la razón matemática que expresa una relación cuantitativa entre la "realidad" y una "representación de la realidad". La escala adquiere sentido educacional, en la medida que se logra resignificar como co-sustancial a lo cotidiano. Muchas veces hemos intentado desde los discursos pedagógico-geográficos, promover la idea de que las escalas mayores de estudio son ajenas a la vivencia de nuestros estudiantes, incluso hemos aceptado, sin mayores resquemores, de que una geografía basada en el estudio de "lo regional" o lo "local" (como sinónimo de una división político-administrativa de la que se es parte), se ajusta a lo potencialmente significante para el estudiante. Incluso se ha intentado asegurar que las escalas menores "como van de lo próximo y concreto" aparecen como apropiadas a ser trabajadas en etapas de desarrollo del pensamiento concreto. A saber, estas afirmaciones no tienen un sólido sustento, en la medida en que se considera que la escala es por sobre todo una referencia a la afectación, siempre presente en la experiencia espacial. Las escalas mayores pueden estar siendo, muchas veces, parte de la experiencia espacial concreta, asimismo las escalas menores podrían pertenecer al plano de lo abstracto en una experiencia espacial. El ejercicio didáctico es acudir indistintamente a las escalas, sin connotar para cada una de ellas un exclusiva etapa de desarrollo cognitiva de los sujetos que participan del acto educativo.

La escala de análisis es un criterio importante en el estudio de la Geografía. Es fundamental que se consideren siempre los varios niveles de esta 
El espacio por aprender, el mismo que enseñar: las urgencias de la educación...

escala social de análisis: lo "local", lo "regional", lo "nacional" y lo "mundial”. (...) El tránsito en los varios niveles de esta escala es fundamental para un análisis significativo y consecuente, de lo contrario existe el riesgo de explicaciones simplistas, que no abarcan todas esas dimensiones y que llevarían a justificar de forma natural (por la naturaleza), problemas que son esencialmente sociales o que derivan de situaciones sociales. (Callai, en Castrogiovanni, 2000, p. 95i)

La escala aparece como resultado de una intencionalidad procedimental, en la medida que interpelo al aprendiz a intentar definir las afectaciones de lo social en la configuración de su experiencia espacial. Todo lo anterior, en el entendido de que el todo aparece fragmentado y que ya no se sabe si se es parte de la totalidad o si se es parte de un fragmento de la misma. La escala como dimensión procedimental, potencia el reconocimiento del otro, en la medida que se definen varianzas de afectación en las experiencias espaciales que concurren en una situación educativa. Lo que para un estudiante aparece como un fenómeno aislado, además puede resultar no ser significativo, mientras que para otro podría ser la manifestación más cruda de su interconexión con lo social. La escala permite constituir un diálogo de las afectaciones, y reconstituir el proyecto de un sujeto que es capaz de ser actor, en la medida que asume aquello "lejano" como cotidiano y propio.

Las diferentes sociedades no sólo producen el espacio, como Lefebvre nos ha enseñado, ellas también producen la escala. La producción de la escala puede ser la diferenciación más elemental del espacio geográfico y es en toda su extensión un proceso social. No hay nada ontológicamente dado sobre la división tradicional entre hogar y localidad, escala urbana y regional, nacional y global. La diferenciación de escalas geográficas establece y se establece a través de la estructura geográfica de interacciones sociales. (Smith, 2002, p. 141)

En este sentido, emerge como prioritario el develamiento de lo normativo que pueden llegar a ser nuestras prácticas pedagógico-geográficas en la medida en que replicamos escalas y fragmentamos la realidad de acuerdo con las apuestas curriculares propuestas a nivel nacional. Con ello, desconocemos la posibilidad que tiene todo individuo de producir escala según sea su experiencia espacial. La escala puede llegar a ser la manifestación más grotesca de nuestra apuesta pedagógica, toda vez que no promovemos el diálogo de las afectaciones particulares, que configuran y dan sentido al yo y al mi experiencial. La escala emerge como un procedimien- 
to que fortalece la dimensión diferencial de las experiencias espaciales, de modo complementario, permite el entendimiento de la totalidad y complejidad de dichas experiencias espaciales. El educador se enfrenta al desafío de acompañar a los sujetos aprendices en la mediación cognitiva entre las distintas graduaciones escalares, para reconstituir la segunda naturaleza de la experiencia espacial.

\section{Lo actitudinal en la experiencia espacial}

Una de las dimensiones más definitorias que conforman la educación del individuo, es la referida al desarrollo de las actitudes. Estas últimas definen al sujeto en si mismo y al actor en relación al otro. Se trata de potenciar pautas de comportamiento asociadas a los proyectos de subjetivación, por un lado y de socialización por otro y que involucran, por lo general, una exteriorización de sensaciones, sentimientos y pensamientos. Lo actitudinal en la educación, actúa normativamente en tanto es pre-existencia de los socialmente aceptable, pero es potencia en términos de su maleabilidad, conforme se establece un diálogo de experiencias entre los protagonistas de la educación. La enseñanza-aprendizaje del espacio permite desarrollar una serie de actitudes en tanto, propicia el entendimiento de la concordancia o disonancia cognitiva de la experiencia espacial que se hace expresión mental-corpórea de los individuos. La experiencia espacial es también una actitud que puede o no coincidir con un "deber ser" actitudinal y que requiere de herramientas pedagógicas para ser interpretadas. Una de dichas herramientas es considerar la conciencia espacial en el entendimiento de la propia vivencia actitudinal. Comprender las actitudes que configuran espacio, es también aprender a leer la exteriorización de mecanismos cognitivos asociados a la percepción y a la representación del mundo. La conciencia es una de esas destrezas que permiten aproximarse al entendimiento de nuestras actitudes. El sujeto perdido y el actor no actuante, necesariamente deben ser acompañados en la definición de formas de conciencia. El enseñante se ve enfrentado a la posibilidad de acompañar al aprendiz en el hacer de si, las construcciones espaciales que le parecen ajenas y que sin embargo han tenido como origen el yo y el nosotros.

La lectura del paisaje es común a cualquier sociedad, en cualquier época. La relación de los hombres con la naturaleza implica niveles de percepción del medio que los abriga. La construcción del hábitat necesariamente involucra 
El espacio por aprender, el mismo que enseñar: las urgencias de la educación...

proyecciones, pre-ideas, evaluaciones, en fin formas de conciencia del espacio. (...) El propio territorio de cierta forma se define en esa autoconciencia. Humboldt desarrolló el concepto de "horizonte geográfico", incluyendo el espacio vivenciado y sus representaciones. (Robert Moraes, 2002a, p. 27)

El horizonte geográfico humboldtiano es muy ilustrativo de la conciencia y de la posibilidad de hacer de si aquello que por algún motivo se encuentra distanciado de mi o de nosotros. Aquello que aparece, en el contexto espacial, como potencialmente propietario es ya una forma de vivenciar y por lo tanto concebirlo como más o menos distante a mi, refiere algún grado de conciencia. La actitud aparece como el deseo propio de hacerlo o no hacerlo para mi, eso es configurar ya, una tierra incógnita que es parte de mi autoconciencia espacial.

Asimismo la conciencia permite situar temporalmente la experiencia espacial en tanto significa el ascenso de un discurso definiendo aquello que es o no geografía. Esto se reafirma al mirar dos de las posibles raíces latinas que tiene la palabra conciencia: "conscendi" que significa subir, ascender y "conscientia" que significa conocimiento compartido. La conciencia es un estado superior de la experiencia que implica el usodesuso de un lenguaje de definición sobre el si mismo y sobre el nosotros. En dicho sentido cobra importancia las aportaciones del profesor Antonio Robert Moraes en tanto nos señala:

Por pensamiento geográfico se entiende un conjunto de discursos a respecto del espacio que sustantivan las concepciones que una determinada sociedad, en un momento determinado, posee acerca de su medio (desde lo local a lo planetario) y de las relaciones con él establecidas. Se trata de un acervo histórico y socialmente producido, una parte de la sustancia de la formación cultural de un pueblo. (Idem, ibid., p. 32)

No hay conciencia prestada. Si queremos lograr un aprendizaje significativo, no podemos instalarlo en una no experiencia. La educación geográfica refiere al espacio vivido y por lo tanto definido y comprendido históricamente con un tipo de discurso sobre el yo y el nosotros. En este contexto, urge recuperar el sentido común de la experiencia espacial y revitalizarlo como la topogénesis de espacios. No hay educación geográfica, más no hay aprendizaje geográfico, sino hemos de acudir permanentemente a dicha topogénesis, procurando de mejor forma avanzar desde nuestros clásicos contenidos cargados de sensacionalismos "aparentemen- 
te" generadores de conciencia geográfica hacia el desarrollo de una conciencia instalada en los proyectos de ser para si y para otros.

Aprender a pensar significa elaborar, a partir del sentido común, del conocimiento producido por la humanidad y de la confrontación con los otros saberes (del profesor, de otros interlocutores), su propio conocimiento. Este conocimiento, partiendo de los contenidos de la Geografía, significa "una conciencia espacial" de las cosas, de los fenómenos, de las relaciones sociales que acontecen en el mundo. (Callai, en Castrogiovanni, 2000, p. 93)

El acto pedagógico se hace ahora, descodificador y un mitificador de los saberes previos, pues de esa forma logra entender las esencias que yacen en las experiencias espaciales cotidianas. Su papel en la concientización no es más que el fortalecimiento de aquello que fue olvidado por las disciplinas escolares. El saber de experiencia hecho de Freire, es un saber igualmente sistemático, igualmente organizado, igualmente (o incluso más) conciente. El saber que deviene de la experiencia espacial informal, es el saber negado de la educación geográfica, tal vez, sea ese saber, el que salve del desmembramiento, de la apatía y del desinterés a los discursos geográficos. El proyecto que se desdibuja en el actual contexto sociocultural, el del sujeto que es actor, no se potencia desde una educación geográfica que desvincula al enseñante y al aprendiz con las manifestaciones de inconformidad y rebeldía respecto de lo que viven (re-mirar obra de Milton Santos). Asimismo no se potencia desde una educación geográfica que no sea entendida como la posibilidad de colaborar con las transformaciones que el mundo requiere. Una buena ilustración de aquello es lo que señala el profesor Nestor Kaercher:

La concientización es penetrar en la realidad, yendo a las esencias de los fenómenos, ultrapasando las simples apariencias. Es un revelar del mundo. Mas ella no es un acto meramente intelectual (...) Siendo la conciencia algo histórico, un proceso a construir, una posibilidad, tenemos otro corolario: que los hombres asuman el papel de sujetos que hacen y rehacen el mundo. (Kaercher, 1997, p. 55-56)

Junto con lo anterior, el desarrollo de la conciencia, como habilidad procedimental, permite avanzar en la construcción de una autonomía moral que permite a su vez, la posibilidad de construir una ética común, en un lenguaje común. La conciencia permite avanzar en la responsabilidad 
El espacio por aprender, el mismo que enseñar: las urgencias de la educación...

social para con los individuos aprendices, al mismo tiempo que permite fisurar aquellos modos pedagógicos que caen en lo pragmático y que niegan la transformación de la realidad:

El enseñante de la Geografía, tal vez con algo más de responsabilidad, sabe de las dificultades y de los beneficios que él asume por su conocimiento de los ambientes educativos. La frágil frontera de transgresión de las éticas se ve enfrentada a las preconcepciones de los enseñantes, el control institucional y a las lógicas valóricas que sustentan el currículum. En dicho escenario se plantea la necesidad de que la práctica pedagógico-geográfica propicie las tomas de conciencia a través de una interacción posibilitadora que tienda a la simetría y que por sobre todo no rompa con las autonomías. La habilidad docente se plantea en la superación de los repertorios pedagógicos encasillados en una forma de hacer instrumental. La confrontación del conocimiento pedagógico con las novedades provenientes de la dinámica educativa, permite que el educador se transforme en un hacedor - con el otro - de la ética en la práctica. (Garrido, 2002, p. 297)

En tal sentido, el presente escrito sólo intenta instalarse en lo progresivo que puede ser el discurso geográfico-educativo, más específicamente, escolar, si se considera que la experiencia espacial informal es a la vez una manifestación del proyecto de sujeto y de actor, negado por las no tradicionales formas emergentes de relacionarse socialmente. La experiencia espacial es un proyecto de subjetividad al mismo tiempo que de socialización, en la medida en que se revaloriza su esencia compleja, más allá de la semantización clásica de espacio como lo contenedor "de". La educación geográfica tiene un metadesafío, el de intentar recuperar dicha experiencia negada y homogeneizada a través de la revitalización del saber cotidiano, de la vivencia diaria y del sentido común. Un intento de aproximación didáctico-pedagógica es el trabajo con categorías de síntesis que permitan develar algunas de esas interrelaciones que configuran un sentido espacial para cada individuo que se encuentra formando parte de un proyecto educacional formal. Asimismo el trabajo con ejes problematizadores permitiría dar cuenta de las fisuras experienciales que los sujetos desarrollan en el intento por educarse en geografía: la potenciación de la interrelación como eje conceptual; la medición escalar como eje procedimental y la conciencia como entendimiento de las actitudes que configuran experiencias.

Recebido em maio de 2005 e aprovado em julho de 2005. 


\section{Referencias bibliográficas}

CALLAI, H. O estudo do lugar e a pesquisa como princípio da aprendizagem. In: Ponencias del 9no EGAL. México: Mérida, 2003.

CASTROGIOVANNI, A. (Org.). Ensino de geografia. Práticas e textualizaçōes no cotidiano. Porto Alegre: Editora Mediação, 2000.

DÜBET, F. Sociologie de l'expérience. Paris: Seuil, 1994.

DÜBET, F.; MARTUCELLI, D. Sociología de la experiencia escolar. Buenos Aires: Losada, 1998.

DÜBET, F.; MARTUCELLI, D. ¿En qué sociedad vivimos?. Buenos Aires: Losada, 1999.

DÜBET, F. et al. Société fragmentée: le multiculturaline en debat. Paris: La Découverte, 1996.

FREIRE, P. La educación como práctica de la libertad. México: Editorial Siglo Veintiuno, 1969.

FREIRE, P. Pedagogía de la esperanza. México: Editorial Siglo Veintiuno, 1993.

FREIRE, P. Politica y educación. México: Editorial Siglo Veintiuno, 1996. GARRIDO, M. La regresión teórica de las investigaciones referidas al quehacer educativo-geográfico en Chile. Anales de la Sociedad Nacional de Ciencias Geográficas, Chile, n. 23, p. 291-300, 2002.

GUSDORF, G. Pourquoi des professeurs?: pour une pédagogie de la pédagogie. París: Payot, 1963.

KAERCHER, N. Desafios e utopias no ensino de geografia. Santa Cruz do Sul: Editora da UNISC, 1997.

MORIN, E. La mente bien ordenada. Barcelona: Editorial Seix Barral, 2000 .

MORIN, E. Introducción al pensamiento complejo. Barcelona: Editorial Gedisa, 1990.

MORIN, E. Los siete saberes necesarios para la educación del futuro. Barcelona: Editorial Gedisa, 2000. 
El espacio por aprender, el mismo que enseñar: las urgencias de la educación...

PULGARÍN, M.R. Enseñanza de las ciencias sociales integradas desde el estudio del espacio geográfico. In: Ponencias del 9no EGAL, México: Mérida, 2003.

ROBERT MORAES, A.; DA COSTA, W. Geografia crítica: a valorização do espaço. São Paulo: Hucitec, 1999.

ROBERT MORAES, A. Ideologias geográficas. São Paulo: Hucitec, 2002a.

ROBERT MORAES, A. Geografia: pequena história crítica. São Paulo: Hucitec, 2002b.

SANTOS, D. A reinvenção do espaço. São Paulo: Editora da Unesp, 2002.

SANTOS, M. Por una geografía nueva. Barcelona: Oikos-Tau, 1990.

SANTOS, M. Território y sociedade (entrevista de Odette Seabre et al.). São Paulo: Editora Fundação Perseu Abramo, 1996 a.

SANTOS, M. De la totalidad al lugar. Barcelona: Oikos-Tau, 1996b.

SANTOS, M. Metamorfosis del espacio habitado. Barcelona: Oikos-Tau, $1996 \mathrm{c}$.

SANTOS, M. La naturaleza del espacio geográfico. Técnica y tiempo; razón y emoción. Barcelona: Ariel Geografía, 2000.

SMITH, N. Geografía, diferencia y las políticas de escala. Terra Livre, n. 19, p. 127-146, 2002.

TUAN, Y.-F. Structuralism, existentialism and environmental perception. Environment and Behavior, v. 3, p 319-331, 1972.

TUAN, Y.-F. Images and mental maps. Annals of the Association of American Geographers, v. 65, p. 205-213, 1975.

TUAN, Y.-F. Geografía humanística. Annals of the Association of American Geographers, v. 66, p. 266-276, 1976.

TUAN, Y.-F. Topofilia. Um estudo da percepção e valores do meio ambiente. São Paulo: DIfEL, 1980.

TUAN, Y.-F. Espaço e lugar: a perspectiva da experiência. São Paulo: DIFEL, 1983. 
TUAN, Y.-F. Landscapes of fear. Oxford: Basil Blackwell, 1979.

TUAN, Y.-F. Escapismo. Formas de evasión en el mundo actual. Barcelona: Editorial Península-Atalaya, 2003. 